\title{
Intermittent intestinal obstruction due to chronic colo-colic \\ intussusception
}

WCG Peh

MBBS, DMRD, FRCR, FAMS

Associate Professor

W Cheng*

MBBS, FRCSE, FRCSG, FRACS

Assistant Professor

Departments of Diagnostic Radiology and *Surgery (Paediatric Surgery Division),

The University of Hong Kong,

Queen Mary Hospital, Hong Kong

\section{Abstract}

A two year old boy

presented with a two

month history of

intermittent bloody

diarrhoea, and

associated intermittent

colicky abdominal

pain and weight loss.

A small mass was

palpable over the left

abdomen. Abdominal

radiograph showed

colonic obstruction

and barium enema

revealed colo-colic

intussusception. At

laparotomy, the

intussusception had reduced

spontaneously and

was found to be

caused by a benign

juvenile polyp. The

clinical and imaging features, and

management of the

chronic form of

intussusception are discussed.

\section{Case report}

A two year old Chinese boy presented with a two month history of intermittent diarrhoea. Mucus and blood was occasionally noted in the stools. There was associated intermittent colicky abdominal pain and weight loss of $2 \mathrm{~kg}$. The child enjoyed previous good health. Prior to admission, he was seen by a private practitioner and had been treated with antibiotics without improvement. On examination, a small firm mass was palpable over the left upper abdomen. Ultrasound scan did not however show any abnormality. Stool cultures for various micro-organisms, and blood serology and cultures, were all negative.

Abdominal radiograph (Figure 1), done during the next severe episode

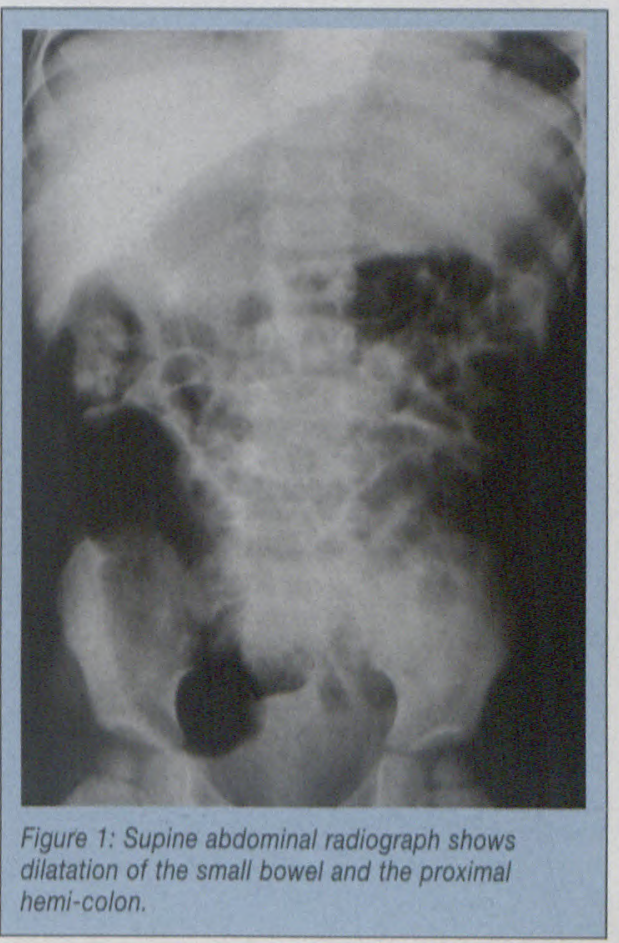

to page 22 


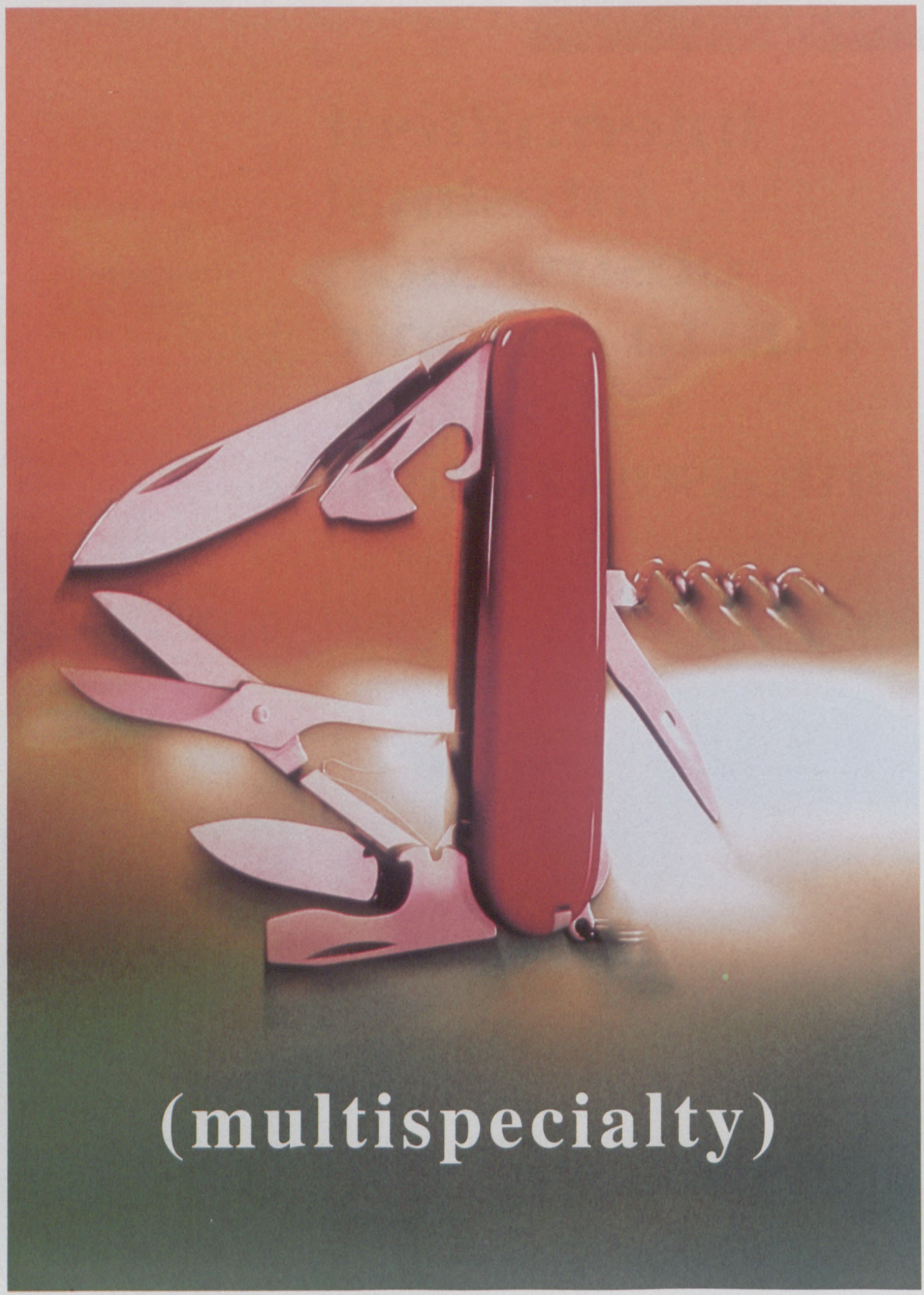




\section{Ready for everything. Announcing ImagePoint, from Hewlett-Packard ${ }^{\oplus}$}

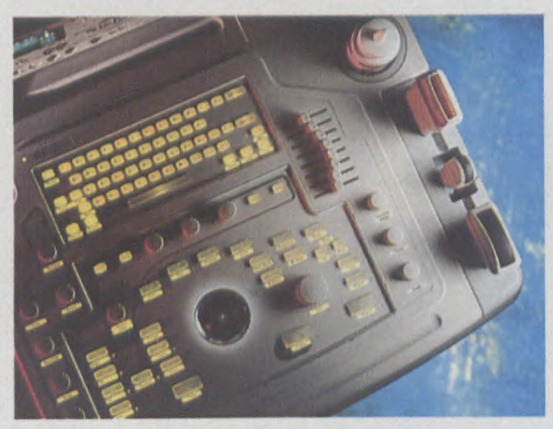

The innovative, ergonomic keyboard is designed for comfort and ease of use.

I magePoint is the multispecialty, high-performance digital ultrasound system that covers a wide range of ultrasound examinations. Whatever your needs - abdominal, vascular, OB/GYN, small parts, cardiac, paediatric - ImagePoint brings Hewlett-Packard's tradition of innovation and performance to ultrasound. And ImagePoint comes complete with all these applications, without compromise in image quality.

ImagePoint's exceptional digital imaging provides outstanding resolution. Its simple, ergonomic design is comfortable and efficient. Global, comprehensive training and support are available when you need them. And ImagePoint is affordable to buy and cost-effective to operate. It's easy to learn, easy to use, easy to upgrade and easy to move.

In short, easy to own.
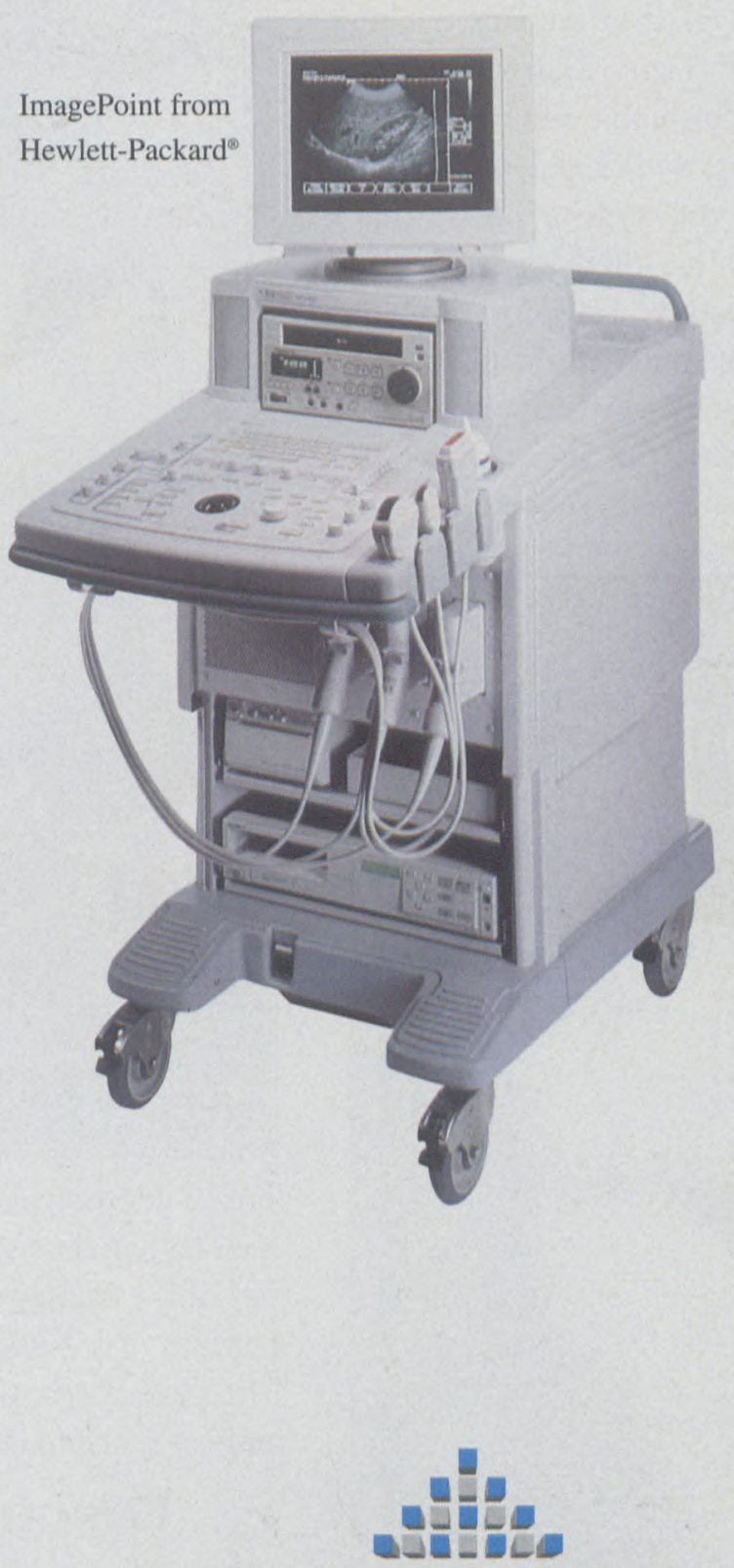

Hiperformance systems

For more information please contact Lynnette Lewis at HiPerformance Systems, sole authorised distributor of Hewlett-Packard medical equipment, at (011) 806-1229 or

Glen Strike in Durban (031) 207-2073 or

Diane Romburgh in Cape Town (021) 658-6202 


\section{Intermittent intestinal obstruction \\ due to chronic colo-colic intussusception}

\section{from page 19}

of abdominal pain, showed dilated loops of gas-filled small bowel, as well as distention of the ascending and transverse colon. An urgent barium enema was performed on suspicion of colonic obstruction in the region of the splenic flexure. Complete obstruction at the proximal descending colon was confirmed, with a concave defect at the head of the barium column and a classic "coiled-spring" appearance, typical of intussusception, beyond that (Figure 2). Combination of plain radiographic and barium enema findings were highly suggestive of the colo-colic type of intussusception. In addition, there was a polypoidal filling defect located just distal to the intussusception site on the barium study (Figure 2). Attempted hydrostatic reduction of intussusception using barium was unsuccessful.

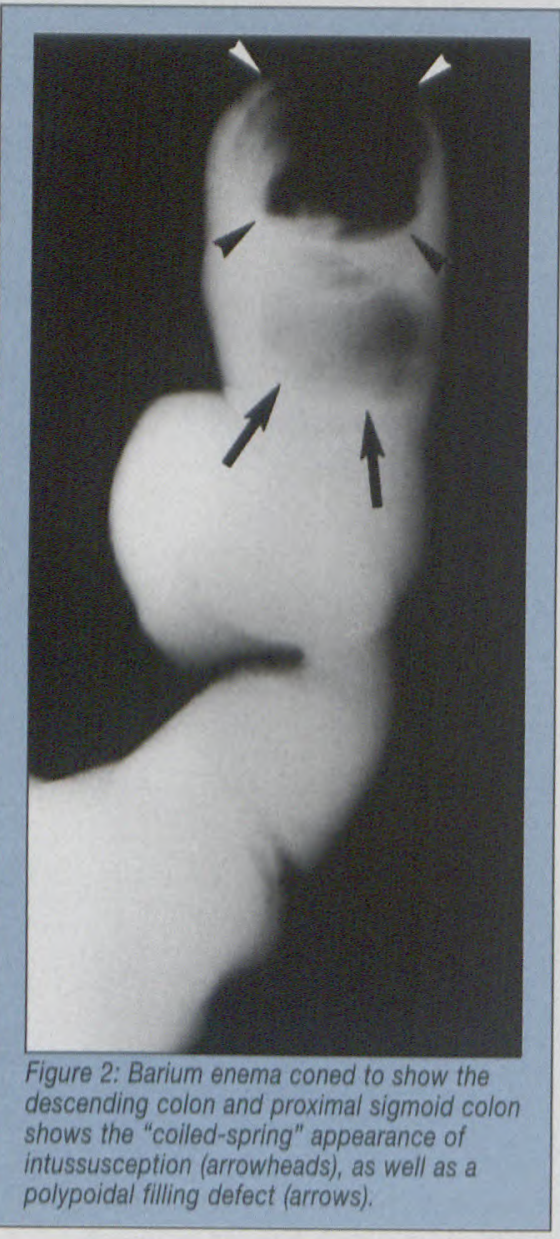

Laparotomy was performed later the same day. At surgery however, the colo-colic intussusception was found to have reduced spontaneously. A mass was palpable within the proximal descending colon and colotomy revealed a $3.5 \times 2.5 \times 1.7 \mathrm{~cm}$ polyp (Figures $3 \mathrm{a}$ and $\mathrm{b}$ ), corresponding in

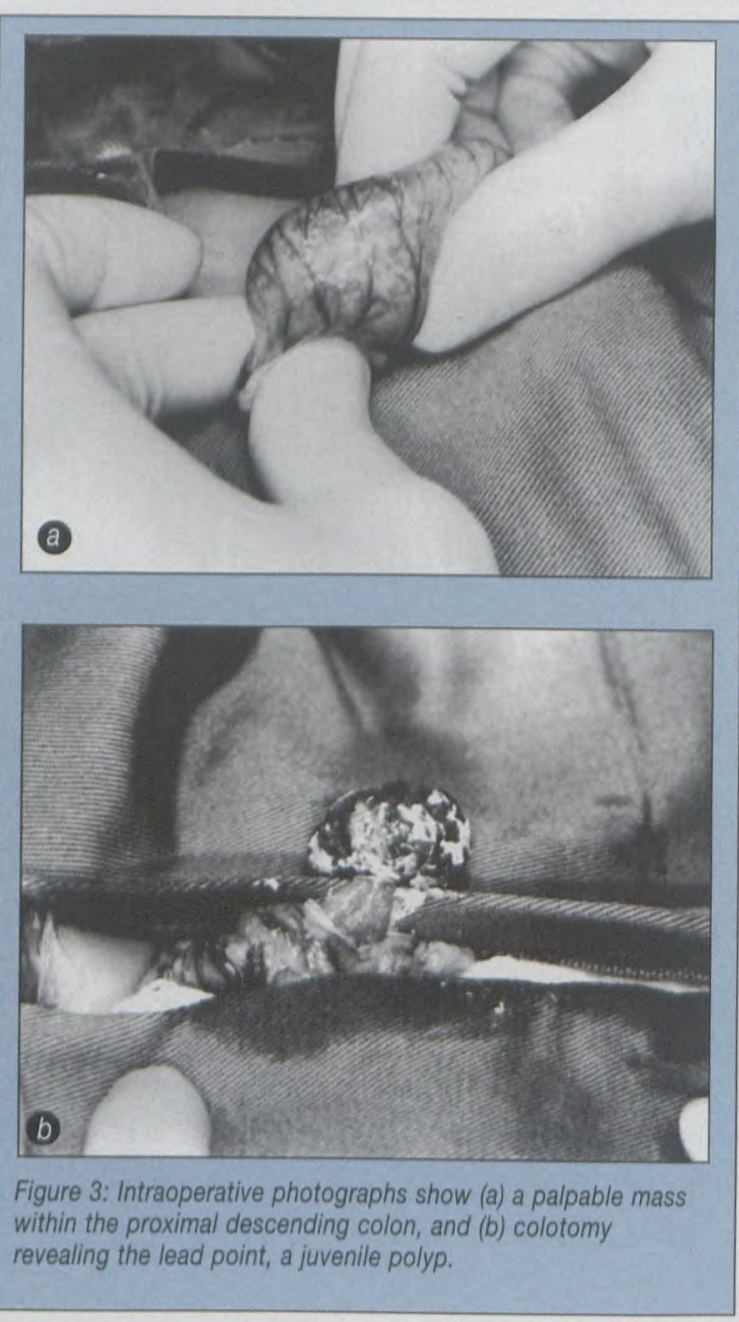

site to the polypoidal filling defect seen on barium enema. Histopathology of the excised specimen showed features of a benign juvenile polyp. The patient recovered uneventfully and has remained well to date.

\section{Discussion}

Intestinal intussusception is a common surgical emergency in children, requiring urgent diagnosis and treatment. Acute intussusception is usually considered early in the differential diagnosis of a young child presenting with a short history of abdominal pain and vomiting. Chronic intestinal intussusception of greater than two weeks duration is uncommon, occurring in $6.5 \%$ of patients in one large series. ${ }^{1}$ Chronic intussusception behaves differently from the acute form in that abdominal pain and vomiting are less severe, with diarrhoea and weight loss being recognized features. Chronic intussusception may even rarely present as failure to thrive. Except for the typical intermittent colicky abdominal pain, symptoms of chronic intussusception are generally rather non-specific. ${ }^{2-4}$ Chronic intussusception tends to be found in older children and it has been suggested that at an older age, the anatomy is such that intussusception occurs without significant impairment of the blood supply. ${ }^{1}$

Ultrasound is now regarded as an accurate method to diagnose intussusception, offering the advantages of being quick, simple, non-invasive and radiation-free. It has been recommended that contrast enema should be reserved for ultrasonically-equivocal cases or for therapeutic reduction. ${ }^{5}$ Spontaneous reduction of intussusception has been reported to occur. ${ }^{6,7}$ On retrospect, the clinical picture of this patient fitted that of a chronic, intermittent-occurring and spontaneously-reducing intussusception. At the 


\section{Intermittent intestinal obstruction due to chronic colo-colic intussusception}

\section{from page 22}

time of ultrasound and at surgery, the intussusceptum was not identified, while in-between, it produced the characteristic barium enema appearances of intussusception.

Colo-colic intussusception is the least frequently encountered type of intussusception, while the ileo-colic type is by far the commonest form. ${ }^{8}$ Although detection of lead points using ultrasound has been reported, ${ }^{9}$ the site of the polyp within a gas- and faecal-filled colon and absence of the spontaneously-reduced intussusceptum probably made ultrasonic assessment difficult in this patient. Causative lead points are not detected in the majority of intussusceptions, being reported in only $2.5-10 \%$ of cases. ${ }^{10,11}$

The presence of barium interposed between the intussusceptum and intussuscipiens, the so-called "dissection sign", could have contributed to failure of hydrostatic reduction in this patient. The "dissection sign" causes concentric compression of the intussusceptum and hence a decrease in the reductive force, due to fluid dynamics of the dissected barium. There is evidence that this sign may be a reliable predictor of failure of hydrostatic reduction. ${ }^{12}$ In any case, even successful reduction of an intussusception does not exclude a lead point. Careful review of all imaging studies and meticulous examination during laparotomy should be carried out to diagnose lead points in order that they may be adequately treated. As chronic intussusception is frequently associated with a predisposing lesion and a low success rate of hydrostatic reduction, early surgical intervention is recommended for this form of intussusception. ${ }^{4}$

\section{References}

1. Reijnen JAM, Festen C, Joosten HJM. Chronic intussusception in children. $\mathrm{Br} J$ Surg. 1989;76:815-816.

2. Page AC, Price JF, Salibury JR, Howard ER, Karani J. Chronic intussusception. Arch Dis Child 1990;65:134-135.

\section{The renal transplant score - a different way of evaluating renal transplant pathology}

\section{from page 18}

2. Dubovsky EV, Russel CD, Erbas B. Radionuclide evaluation of renal transplants. Seminars in Nuclear Medicine 1995; 25: 4959 .

3. Nicoletti R. Evaluation of renal transplant perfusion by functional imaging. Eur J Nucl Med 1990; 16: 733-739

4. Mizuiri S, Hayoshi I, Takano M, Ban R, Ohara T, Susiki Y, Hasegawa A. Fractional mean transit time in transplanted kidneys studied by technetium-99m-DTPA Comparison of clinical and biopsy findings. J Nucl Med 1994; 35: 84-89.
5. Ash J, De Souza M, Peters M, Wilmont D, Hansen D, Gilday D. Quantitative assessment of blood flow in Pediatric recipients of renal transplants. $J$ Nucl Med 1990; 31: 580-585.

6. Dubovsky EV, Russel CD. Radionuclide evaluation of renal transplants. Seminars in Nuclear Medicine 1988; 18(3): 181-198.

7. Kittner $\mathrm{CH}$, Esther G, Finck W, Bast R, Templin R, Wizze H, Brockmöller S. Der Trendverteilungs Quotient, ein neues Werkzeug zur Auswertung der Nierenperfusionsszintigrafie mit $99 \mathrm{~m}-\mathrm{Tc}$ DTPA nach Nieren Transplantation. Nucl Med 1993; 32: 37-41.
3. Watson NA, Bisset RAL. Intussusception - a cause of chronic abdominal symptoms and weight loss. Clin Radiol 1994;64:723-726.

4. Rees BI, Lari J. Chronic intussusception in children. Br J Surg 1976;63:33-35.

5. Shanbhogue RLK, Hussain SM, Meradji M, Robben SGF, Vernooij JEM, Molenaar JC. Ultrasonography is accurate enough for the diagnosis of intussusception. J Pediatr Surg 1994:29:324-328

6. Morrison SL, Stork E. Documentation of spontaneous reduction of childhood intussusception by ultrasound. Pediatr Radiol 1990;20:358-359

7. Swischuk LE, John SD, Swischuk PN. Spontaneous reduction of intussusception: verification with ultrasound. Radiology 1994;192:269-271.

8. Bissett GS III, Kirks DR. Intussusception in infants and children: diagnosis and therapy. Radiology 1988;168:141-145

9. Lam AJ, Firman K. Ultrasound of intussusception with lead points. Australas Radiol 1991;35:343-345.

10. Ein SH. Leading points in childhood intussusception. J Pediatr Surg 1976; 11:209211

11. Miller SF, Landes AB, Dautenhahn LW, et al. Intussusception: ability of fluoroscopic images obtained during air enemas to depict lead points and other abnormalities. Radiology 1995;197:493-496

12. Fishman MC, Borden S, Cooper A. The dissection sign of nonreducible ileocolic intussusception. AJR 1984;143:5-8.
8. Lear JL, Raff U, Jain R, Horgen JG Quantitative measurement of renal perfusion following transplant surgery. $J$ Nucl. Med. 1988; 29: 1656-1661.

9. Smith SB, Wombolt DG. Histologic correlation of transplant rejection diagnosed by computer assisted sulphur colloid scan. Urology 1983; 21(2): 151-153.

10. Kim EE, Pjura G, Lowry P, Verani R, Sandler C, Flechner S, Kahan B. Cyclosporin - A nephrotoxicity and acute cellular rejection in renal transplant recipients: correlation between Radionuclide and Histologic findings. Radiology 1986; 159: 443-446. 